\title{
DIAGNÓSTICOS DE ENFERMAGEM EM MULHERES QUE FREQÜENTAM SERVIÇO DE PLANEJAMENTO FAMILIAR
}

Gislaine E. Kuahara Camiá*

Heimar de Fátima Marin**

Márcia Barbieri**

Camiá GEK, Marin HF, Barbieri M. Diagnósticos de enfermagem em mulheres que freqüentam serviço de planejamento familiar. Rev Latino-am Enfermagem 2001 março; 9(2): 26-34.

Este trabalho teve como objetivo identificar os diagnósticos de enfermagem em mulheres matriculadas em um serviço de planejamento familiar. Foi elaborado um instrumento de coleta de dados segundo os Padrões Funcionais de Saúde ${ }^{(8-9)}$. A amostra foi constituída por 100 mulheres adultas. Identificou-se 49 diagnósticos de enfermagem, sendo 9 com freqüência igual ou superior a 45,0\%. Ressalta-se a importância da realização de outros estudos em instituições que ofereçam assistência em planejamento familiar, no intuito de observar se os diagnósticos de enfermagem encontrados na população do referido serviço podem ser extensivos a população geral.

UNITERMOS: enfermagem, diagnóstico de enfermagem, planejamento familiar

\section{NURSING DIAGNOSES IN WOMEN ATTENDING A FAMILY PLANNING PROGRAM}

The purpose of this study was to identify nursing diagnoses in women enrolled in a family planning program. An instrument of data

collection was elaborated according to the Functional Health Patterns ${ }^{(8-9)}$. The sample consisted of 100 adult women. Forty-nine nursing diagnoses, of which 9 with a frequency equal or higher to 45,0\% were identified. Further studies are still needed in institutions that offer assistance in the area of family planning in order to examine if the nursing diagnoses found in these women can be generalized to the population.

KEY WORDS: nursing diagnoses, family planning

\section{DIAGNÓSTICOS DE ENFERMERÍA EN MUJERES QUE FRECUENTAN EL SERVICIO DE PLANIFICACIÓN} FAMILIAR

Este trabajo tuvo como objetivo identificar los diagnósticos de enfermería en mujeres matriculadas en el servicio de planificación familiar. Un instrumento de recolección de datos fue elaborado conforme los Patrones Funcionales de la Salud ${ }^{(8-9)}$. La muestra consistió de 100 mujeres adultas. Se identificaron 49 diagnósticos de enfermería, de los cuales 9 con frecuencia igual o superior a 45,0\%. Se resalta la importancia de la realización de otros estudios en instituciones que ofrezcan asistencia en el planificación familiar con el objetivo de observar si los diagnósticos de enfermería encontrados en la población del referido servicio pueden ser extendidos a la población en general.

TÉRMINOS CLAVES: enfermería, diagnóstico de enfermería, planificación familiar

\footnotetext{
* Enfermeira Obstetra, Mestre em Enfermagem pela Universidade Federal de São Paulo/Escola Paulista de Medicina (UNIFESP/EPM). Professor Adjunto da UNICSUL e UNIP. Endereço: Rua: Itapiru, 281 - Ap. 111 - Saúde - 04143-010 - São Paulo - SP - Brasil

**Professor Adjunto da Disciplina Enfermagem Obstétrica da Universidade Federal de São Paulo/Escola Paulista de Medicina (UNIFESP/EPM)
} 


\section{INTRODUÇÃO}

$\boldsymbol{O}$ planejamento familiar deve ser um elemento essencial na prevenção primária de saúde, auxiliando as pessoas que procuram tais serviços, oferecendo-lhes informações necessárias para a escolha e uso efetivo dos métodos anticoncepcionais que melhor se adaptem às condições atuais de saúde. 0 enfermeiro encontra-se em uma posição impor tante para auxiliar os casais a compreenderem as opções de métodos contraceptivos disponíveis ${ }^{(1)}$.

Algumas pesquisas revelam que, quanto melhor for a qualidade da orientação prestada, maior será a adequação na escolha, satisfação, aceitabilidade e continuidade no uso do método anticoncepcional, o que ocasionará diminuição no índice de falha ${ }^{(2-}$ 3).

Na prática clínica em planejamento familiar, é importante a utilização do Processo de Enfermagem, concretizado através da consulta de enfermagem, que é um recurso utilizado pelo enfermeiro para que o mesmo possa atuar de maneira independente e direta junto ao cliente.

A primeira etapa do processo de enfermagem, ou seja, o histórico, levantamento de dados ou avaliação inicial do paciente, compreende a entrevista de enfermagem, exame físico, resultados laboratoriais e outras fontes, com o propósito de identificar as necessidades, problemas, preocupações ou reações humanas do cliente $^{(4)}$.

Após análise e interpretação dos dados, os diagnósticos de enfermagem são identificados propiciando um foco central para as fases subseqüentes. É importante ressaltar que, estes não podem ser retirados do contexto global que caracteriza o Processo de Enfermagem, pois resultariam em intervenções inapropriadas ${ }^{(5)}$. Esta fase oferece um método eficiente de comunicação dos problemas do cliente e baseando-se nesta etapa, o plano de cuidados é elaborado, implementado e avaliado ${ }^{(4)}$.

0 diagnóstico de enfermagem é importante para a profissão e para o cliente, pois direciona o cuidado possibilitando uma assistência individualizada. É definido pela North American Nursing Diagnosis Association - NANDA ${ }^{(6)}$, como um "julgamento clínico das respostas do indivíduo, família ou da comunidade aos processos vitais ou aos problemas de saúde atuais ou potenciais. Fornece a base para a seleção das intervenções de enfermagem para atingir os resultados pelos quais o enfermeiro é responsável".

Embora a classificação de diagnósticos da NANDA seja, de certo modo, conhecida e divulgada, ela é uma das muitas já existentes. A existência de diferentes sistemas de classificação desenvolvidos para prática de enfermagem surgiu devido a necessidade de um registro único dos dados, garantindo que no prontuário do paciente, os dados de enfermagem estejam presentes e possam ser analisados, mostrando a contribuição de enfermagem na área da saúde ${ }^{(7)}$. Segundo esta mesma autora, com o advento da era da informática, fez-se necessário definir um padrão de vocabulário que fosse capaz de descrever o quê os enfermeiros fazem, como fazem e que resultados conseguem obter decorrentes da ação.

Neste estudo, foi utilizado o Sistema de Classificação dos Padrões Funcionais de Saúde ${ }^{(8-9)}$, precursor do sistema NANDA. A diferença entre eles é que o primeiro denomina seus padrões de Tipologia e não de Taxonomia. A autora adverte que seu sistema é para avaliar um cliente, cuja estrutura é composta por onze Padrões Funcionais de Saúde. São eles: Percepção da saúde/manutenção da saúde; Nutricional/metabólico; Eliminação; Atividade/exercício; Sono/repouso; Cognitivo/perceptivo; Autopercepção/autoconceito; Papel/relacionamento; Sexualidade/reprodução; Enfrentamento/ tolerância ao estresse e Valor/crença ${ }^{(8-9)}$.

\section{OBJETIVO}

Considerando que esta etapa do processo de enfermagem pode favorecer estratégias para elevar a qualidade das ações profissionais oferecidas e contribuir para o fortalecimento da estrutura de conhecimento na área, decidiu-se identificar, neste trabalho, os diagnósticos de enfermagem mais freqüentes, apresentados nas mulheres de um serviço de planejamento familiar.

\section{METODOLOGIA}

0 estudo foi realizado no Serviço de Planejamento Familiar da Universidade Federal de São Paulo (UNIFESP), localizado na cidade de São Paulo, após solicitação prévia ao profissional responsável e aprovação, bem como pela Comissão de Ética Médica da Universidade.

A amostra foi constituída por 100 mulheres adultas, com idade igual ou superior a 20 anos, que concordaram em participar voluntariamente do estudo, após explicação do trabalho que seria desenvolvido e que, estavam presentes no serviço para a primeira consulta, no período de 29 de junho a 15 de setembro de 1998, representando um percentual de $85,5 \%$ das mulheres atendidas neste período.

0 instrumento de pesquisa utilizado para a identificação dos diagnósticos de enfermagem foi elaborado pelas autoras, baseando-se nos Padrões Funcionais de Saúde, direcionado para atenção primária em planejamento familiar, no intuito de atender a cliente nos aspectos biológico, psicológico, social, cultural e espirituall $^{(8-9)}$. 
No intuito de facilitar a implantação do instrumento de coleta de dados na prática assistencial diária e visando a futura informatização em um sistema de apoio à decisão, optou-se por elaborar, preferencialmente, questões fechadas, enquadrando os possíveis diagnósticos de enfermagem em cada padrão funcional. Vale ressaltar porém que, para elaborar um diagnóstico de enfermagem, são necessários vários dados, que provavelmente não estarão contidos necessariamente em apenas um padrão funcional. Via de regra, o uso global de todos os dados levantados permite, através do julgamento clínico, identificar um diagnóstico específico, cujos dados estavam presentes, originalmente, em um ou mais padrões funcionais. Assim, o instrumento apresenta algumas questões abertas, com espaços disponíveis para documentar os diagnósticos listados ao lado, que podem não estar presentes. Além disso, o padrão que compreende dados sobre Sexualidade e Reprodução foi intencionalmente o mais explorado, por tratar-se de um estudo realizado na área de planejamento familiar.

0 instrumento elaborado consta inicialmente dos seguintes dados:

- de identificação da cliente, como o nome, idade, cor, escolaridade, estado civil, união consensual ou não, procedência, profissão e/ou ocupação;

- do cônjuge, sendo incluídos dados sobre a idade, profissão e/ou ocupação, escolaridade e procedência. Foi considerado cônjuge também aquele que vivia maritalmente;

- da habitação, número de cômodos da casa e se a disposição afetava a privacidade do casal;

- sócio-econômicos, a renda familiar e a existência de recursos financeiros para freqüentar o serviço de planejamento familiar e dar continuidade ao uso do contraceptivo escolhido e tratamento prescrito.

Na seqüência do instrumento, foram inseridos dados referentes aos Padrões Funcionais de Saúde de Gordon, mas direcionados para a área em estudo:

- Percepção/manutenção da saúde, o motivo da vinda ao planejamento familiar, antecedentes pessoais e familiares, além de hábitos e comportamentos da cliente e do cônjuge;

- Nutricional/metabólico, dados sobre a alimentação, aumento e/ou perda de peso no último ano, estatura e o peso atual para o cálculo do índice de massa corpórea, tendo como referência a classificação da Organização Mundial de Saúde ${ }^{(10)}$;

- Eliminação, dados sobre a evacuação, micção, perspiração/ sudorese e eliminação vaginal;

- Atividade/exercício, foram listadas apenas quatro atividades da vida diária, das dez existentes ${ }^{(9)}$, pois a maioria das clientes atendidas no serviço de planejamento familiar não possui quase nenhum problema de limitação física. Porém, algumas clientes possuíam seqüelas, decorrentes de acidente vascular cerebral, por exemplo, ou outras patologias. Neste padrão, foram também incluídos o lazer e o esporte;

- Sono e repouso, dados que possibilitassem investigação e observação de sinais de cansaço e presença de insônia, aspectos importantes a serem observados na mulher;

- Cognitivo/perceptivo, dados que permitissem investigar se a cliente havia entendido as explicações sobre os métodos contraceptivos existentes, fornecidas durante a aula que antecedia a primeira consulta. Nesse momento, era realizada uma revisão de todos os métodos em caso de dúvidas. Todos os órgãos dos sentidos também foram incluídos neste item, além de queixas de dor e desconforto; - Auto-percepção e auto-conceito, dados da imagem do corpo, percepção da cliente sobre si e auto-estima presentes;

- Papel e relacionamento, itens sobre as relações entre a cliente e o parceiro, a família, os amigos e os filhos, além da existência de violência;

- Sexualidade/reprodução, fatores importantes para a área em estudo foram abordados, como por exemplo: menarca, menstruação atual (intervalo, duração, data da última menstruação-DUM), dismenorréia, tensão pré-menstrual (TPM), número de parceiros atuais e anteriores, freqüência e início das atividades sexuais, dispareunia, libido, orgasmo, número de gestações, partos e abortos. Também estão presentes questões sobre 0 auto-exame das mamas e sobre o exame cérvico-uterino (Papanicolaou). Quanto aos métodos contraceptivos, questões como a utilização anterior e atual de algum método, a escolha da cliente e a participação do companheiro neste processo, foram dados essenciais para 0 planejamento da assistência individualizada;

- Enfrentamento/tolerância ao estresse, a reação da cliente diante de um problema conjugal e de uma crise;

- Valor/crença, dados sobre a religião e a interferência desta, na escolha do método contraceptivo.

Os dados do exame físico foram inseridos nos Padrões Funcionais, viabilizando a construção de um instrumento único que respeitasse o modelo adotado na prática de enfermagem.

No final do instrumento, houve espaço para o registro do parecer do enfermeiro, para o profissional documentar o resumo de suas principais conclusões, bem como as intervenções, encaminhamentos e exames solicitados.

A literatura de referência básica, utilizada como suporte na identificação dos diagnósticos de enfermagem mais incidentes na amostra populacional foi o Manual de Diagnósticos de Enfermagem ${ }^{(9)}$, que também contempla as categorias diagnósticas da NANDA ${ }^{(6)}$ e o modelo de assistência proposto ${ }^{(5)}$.

Optou-se por apresentar os diagnósticos de enfermagem com freqüência igual ou superior a $45,0 \%$, demonstrando suas características definidoras e/ou fatores de risco e fatores relacionados. 
RESULTADOS E DISCUSSÃO

Foram identificados 49 diagnósticos de enfermagem, dos quais 45 estão listados na Tabela $1^{(6,9)}$. Quatro diagnósticos restantes foram sugeridos ${ }^{(5)}$. São eles: Risco para Transmissão de Infecção, Risco para Função Respiratória Alterada, Risco para Perfusão Tissular Periférica Alterada e Conforto Alterado.

Tabela 1 - Mulheres adultas atendidas no serviço de planejamento familiar segundo os diagnósticos de enfermagem. UNIFESP/EPM. São Paulo, SP 1998

\section{DLڤGHÓSTICOS DE EHFERHڤGEH}

1. Comportamentos para elevar o nivel de saúde

2. Potencial para aumento do bem-estar espiritual

3. Manutenção da saúde atterada

4. Distúrbio no padrão do sono

5. Risco para controle ineficaz do regime terapêutico

6. Risco para infecção

7. Padrões de sexualidade alterados

8. Estratégias ineficazes de resolução individual

9. Nutrição alterada: ingestão maior do que as necessidades corporais

10. Conforto alterado

11. Medo

12. Déficit de conhecimento

13. Risco para perfusão tissular perfíérica alterada

14. Risco para trauma

15. Integridade tissular prejudicada

16. Déficit de lazer

17. Distúrbio na imagem corporal

18. Processos familiares alterados

19. Ansiedade

20. Risco para função respiratónia alterada

21. Constipação colônica

22. Mucosa oral alterada

23. Integridade da pele prejudicada

24. Constipação percebida

25. Manutenção do lar prejudicada

26. Risco para transmissão de infecç̃åo

27. Risco para déficit de volume de líquidos

28. Nutrição alterada: risco para ingestão maior do que as necessidades corporais

29. Intolerância à atividade

30. Interação social prejudicada

31. Padrões de eliminação urinário alterados

32. Incontinência por pressão

33. Angústia espiritual

34. Risco para solidão

35. Não comprometimento

36. Risco para integridade da pele prejudicada

37. Disfunção na reação de pesar

38. Baixa auto-estima

$3 \quad 3,0$

39. Impotência

40. Nutrição alterada: ingestão menor do que as

$3 \quad 3,0$
necessidades corporais

41. Fadiga

42. Comunicação verbal prejudicada

43. Conflito de decisão

44. Reação de pesar antecipada

45. Senso-percepção alterada

46. Conflito no desempenho de papéis dos pais

47. Risco para desgaste do papel do cuidador

48. Processos de pensamentos atterados

49. Déficit no auto cuidado: banho/higiene

\section{FREQQÜÊHCK}

n

$100 \quad 100,0$

$93 \quad 93,0$

$68 \quad 68,0$

$66 \quad 66,0$

$53 \quad 53,0$

$51 \quad 51,0$

$48 \quad 48,0$

$48 \quad 48,0$

$47 \quad 47,0$

44

44,0

38,0

36,0

36,0

34,0

33,0

32,0

29,0

28,0

26,0

26,0

25,0

20,0

18,0

12,0

10,0

8,0

8,0

7,0

6,0

6,0

5,0

4,0

4,0

4,0

4,0

4,0

3,0

Os diagnósticos de enfermagem, com freqüência igual ou superior a 45,0\%, são listados a seguir:

\section{Comportamentos para elevar o nível de saúde}

Este diagnóstico não possui fatores relacionados e por este motivo, serão demonstradas as características definidoras que sustentam este diagnóstico ${ }^{(6,9)}$. Estas mesmas fontes literárias recomendam que ele seja especificado. Assim, o diagnóstico Comportamentos para Elevar o Nível de Saúde está relacionado ao tema planejamento familiar de uma forma isolada e em associação com outras atividades como auto-exame das mamas, exame cérvicouterino (Papanicolaou), programa de exercícios regulares e abandono de práticas não-saudáveis.

0 diagnóstico de enfermagem Comportamentos para Elevar o Nivel de Saúde, foi identificado na totalidade das clientes entrevistadas, no que se refere ao planejamento familiar. Todas foram incluídas neste diagnóstico por entender que as mulheres que procuraram o serviço apresentaram um "desejo expresso para buscar um nível mais elevado de bem-estar" e "aumentar as práticas de controle de saúde". Ainda, todas as mulheres relataram que estavam no local para evitar a gravidez, escolher um método contraceptivo, realizar acompanhamento do anticoncepcional em uso, realizar exame físico e laboratorial ou receber orientações sobre métodos anticoncepcionais.

Também encontraram este mesmo diagnóstico de enfermagem para o planejamento familiar, pois as clientes procuram o serviço para prevenir a gravidez indesejada, que pode trazer conseqüências físicas e emocionais desagradáveis ${ }^{(11)}$.

0 diagnóstico de enfermagem Comportamentos para Elevar o Nível de Saúde é melhor formulado como uma afirmativa diagnóstica, em uma prática de saúde almejada, especificada ${ }^{(5)}$. Portanto, apesar de ser um diagnóstico único, é sustentado por 
atividades realizadas pelas mulheres estudadas, que são descritas a seguir.

Neste diagnóstico as atividades que caracterizaram a formulação foram:

- realização do auto-exame das mamas: 27,0\% das mulheres relataram a prática mensal. As demais, o faziam de forma esporádica ou não realizavam este exame preventivo.

- realização do exame cérvico-uterino (Papanicolaou): 76,0\% das mulheres realizavam o exame anualmente. Algumas mulheres já estavam informadas que na primeira consulta seriam submetidas a este exame. As 76 mulheres respondentes $(76,0 \%)$ estavam com o exame atualizado ou haviam realizado há no máximo um ano atrás. - realização de programas de exercícios regulares: 10,0\% das clientes relataram esta prática. Tal atividade é importante por evitar o sedentarismo que pode predispor a diversas patologias.

- abandono de práticas não saudáveis, tais como fumo, foi relatado por $8,0 \%$ das entrevistadas. Algumas clientes que eram tabagistas, relataram que abandonaram esta prática, mas que às vezes pensavam em retornar porque haviam adquirido peso.

\section{Potencial para aumento do bem-estar espiritual}

0 diagnóstico de enfermagem Potencial para o Aumento do Bem-estar Espiritual é definido como sendo o processo de desenvolvimento do indivíduo revelando o mistério através da interligação harmoniosa com a fonte da força interior ${ }^{(9)}$. Este diagnóstico foi recebido pela NANDA para desenvolvimento ${ }^{(9)}$ e por ser um diagnóstico positivo, não possui fatores relacionados ${ }^{(5)}$.

Entre as cem mulheres entrevistadas, 93 apresentaram este diagnóstico, expressando que possuíam uma religião. Embora as doutrinas religiosas recomendam a abstinência sexual fora do casamento e o não uso de métodos contraceptivos, ao interrogálas se a religião influenciava na escolha do anticonceptivo, apenas quatro clientes responderam positivamente.

\section{Manutenção da saúde alterada}

0 diagnóstico de enfermagem Manutenção da Saúde Alterada é aplicável tanto às populações sadias quanto às doentes, que expressam o desejo de modificar um estilo de vida pouco saudável ${ }^{(5)}$. Da mesma maneira que o diagnóstico de enfermagem Comportamentos para Elevar o Nível de Saúde, o diagnóstico Manutenção da Saúde Alterada deverá ser especificado, voltado para a prática almejada.

Tabela 2 - Características definidoras identificadas para o diagnóstico de enfermagem Manutenção da Saúde Alterada. Serviço de Planejamento Familiar da UNIFESP/EPM. São Paulo, SP 1998

\section{CARATERISTICAS DEFIHIDORAS}

Inabilidade observada ou relatada para assumir responsabilidade de atender práticas básicas de saúde, $68 \quad 100,0$ em uma ou todas as áreas dos padrões funcionais

História de falta de comportamento em busca da saúde

Falta demonstrada de conhecimento sobre as práticas básicas de saúde

$\mathrm{Na}$ Tabela 2, são demonstradas as características definidoras para o diagnóstico de enfermagem Manutenção da Saúde Alterada. Foram evidenciadas a não realização de práticas como auto-exame das mamas, exame cérvico-uterino e práticas saudáveis, ou seja, as atividades que, por outro lado, explicaram o diagnóstico de enfermagem Comportamentos para Elevar o Nível de Saúde. Os fatores relacionados para este diagnóstico estão listados na Tabela 3.

Tabela 3 - Fatores relacionados ao diagnóstico de enfermagem Manutenção da Saúde Alterada. Serviço de Planejamento Familiar da UNIFESP/EPM. São Paulo, SP 1998

\section{FTORES RELACIOHADOS}

\section{$\mathrm{N}=68$ FREQTî̈EHCK}

Etapas do desenvolvimento não atingidas n $\$$ $\begin{array}{llll}\text { Falta observada ou relatada de recursos materiais } & 53 & 77,9\end{array}$ $\begin{array}{llll}\text { Estratégias ineficazes de resolução } & 48 & 70,6\end{array}$ Falta observada ou relatada de sistemas de apoio pessoal $26 \quad 38,2$ $\begin{array}{llll}\text { Experiência de pesar } \quad 4 & 5,9\end{array}$ Falta ou atteração nas habilidades de comunicação $\quad 2 \quad 2,9$ Dificuldade perceptual ou cognitiva

0 fator relacionado "etapas do desenvolvimento não atingidas" foi identificado em 68 mulheres $^{(6,9)}$.

Para melhor compreensão deste fator, buscou-se mais detalhes de informação nas publicações onde a autora explica 0 fator relacionado, baseando-se em dados relativos à idade, também denominados de maturacionais, como o controle de peso com boa nutrição, dieta baixa em colesterol, aconselhamento sobre 0 planejamento familiar, as práticas sexuais, a importância do exercício regular, as conseqüências do uso de álcool e drogas, auto-exame mensal das mamas, mamografia anual após os 35 anos, investigação do câncer cérvico-uterino a cada ano, entre outros ${ }^{(5)}$.

\section{Distúrbio no padrão do sono}

0 comprometimento do sono está entre as queixas clínicas 
mais freqüentes. Estima-se que um terço da população de adultos experimenta os distúrbios ocasionais ou persistentes do sono ${ }^{(12-}$ 13).

A "queixa verbal de não sentir-se bem repousado" é a característica principal deste diagnóstico de enfermagem, sendo representada pela maioria das entrevistadas.

A maior freqüência de fatores relacionados ao diagnóstico de enfermagem Distúrbio no Padrão do Sono foi o "estresse familiar" com 66 respostas (Tabela 4). Para a inclusão neste item foram considerados todos os fatores pertinentes que poderiam interferir no sono da cliente. Foram detectados problemas com filhos e/ou doença na família e desentendimentos com o companheiro, por motivos diversos, em especial o desemprego, que acarretava 0 estresse familiar.

\section{Tabela 4 - Fatores relacionados ao diagnóstico de enfermagem Distúrbio no Padrão do Sono. Serviço de Planejamento Familiar da UNIFESP/EPM. São Paulo, SP 1998}

\section{FNORES RELACIOHDOS}

Estresse familiar
Enfado diurno
Desconforto físico
Mudanças de hábito ou ambiente
Ansiedade
Nictúria
Medo

5. Risco para controle ineficaz do regime terapêutico

0 diagnóstico de enfermagem Risco para Controle Ineficaz do Regime Terapêutico é definido como a presença de fatores de risco para dificuldades na regulação/integração a um programa de tratamento ou prevenção na vida diária ${ }^{(9)}$.

"Dificuldades econômicas" foi o principal fator de risco para este diagnóstico. As mulheres verbalizaram que não teriam condições financeiras para comprar contraceptivo prescrito ou outro medicamento qualquer, se estes não fossem oferecidos gratuitamente pelo serviço de planejamento familiar. Algumas relataram que teriam problemas para freqüentar o serviço se as consultas subsequentes não fossem marcadas próximas das datas do pagamento de seus salários, pois não teriam recursos financeiros para o transporte.

Segundo alguns estudos, as condições econômicas influenciam significativamente a escolha e o uso dos métodos contraceptivos $^{(14-15)}$.

A "fadiga" (86,8\%) e a "depressão" (54,7\%) podem também representar riscos para a não utilização dos contraceptivos, principalmente aqueles que necessitam de continuidade, como os hormônios orais que, se for esquecida a ingestão diária, não apresentarão a eficácia desejada.

0 fator de risco "conflito familiar" apresentou 52,8\% para a escolha dos métodos contraceptivos. 0 "déficit de conhecimento" foi observado em $39,6 \%$, quando as mulheres verbalizavam que não entenderam o que era o diafragma, como era inserido 0 dispositivo intra-uterino (DIU), como era utilizada a pílula e se 0 hormonal injetável era confiável.

A "complexidade do regime terapêutico" (18,9\%), foi detectada por relatos de dificuldades no uso da pílula e esquecimento.

0 "apoio social deficiente" como fator de risco apresentou um percentual de $15,1 \%$. Segundo o relato das clientes, os homens não gostam de utilizar o condom. Assim, pode-se inferir que algumas mulheres ficam expostas a doenças sexualmente transmissíveis e gravidez indesejada, devido a falta de apoio dos companheiros no que se refere à contracepção.

\section{Risco para infecção}

0 diagnóstico de enfermagem Risco para Infecção é definido pela presença de risco aumentado para a invasão de organismos patogênicos ${ }^{(9)}$. Outros autores ${ }^{(11,16)}$ também encontraram este mesmo diagnóstico em estudos realizados em planejamento familiar.

Alguns métodos contraceptivos predispõem a maiores riscos de infecção do que outros e, por este motivo, deverão ser avaliados os fatores individuais e situacionais relacionados aos seus usuários $^{(17)}$.

Tabela 5 - Fatores de risco para o diagnóstico de enfermagem Risco para Infecção. Serviço de Planejamento Familiar da UNIFESP/EPM. São Paulo. SP 1998

\section{FATORES DE RISCO}

$$
\begin{gathered}
N=51 \\
\text { FREQIÜEHCK }
\end{gathered}
$$

Conhecimento insuficiente para evitar a exposição aos $51 \quad 100,0$ patógenos

Presença de estressores

Estase de líquidos ou secreções do corpo

Destruição de tecidos

Solução de continuidade da pele

Doenças crônicas com debilidade

Imunossupressão

Desnutrição

$51 \quad 100,0$

$33 \quad 64,7$

$23 \quad 45,1$

$11 \quad 19,6$

$4 \quad 7,8$

$4 \quad 7,8$

$2 \quad 3,9$

\section{Padrões de sexualidade alterados}

0 diagnóstico de enfermagem Padrões de Sexualidade Alterados é definido como sendo a expressão de preocupação relativa a sexualidade ${ }^{(9)}$. 
A característica definidora para este diagnóstico é o relato de dificuldades, limitações ou mudanças de comportamentos ou atividades sexuais. Este diagnóstico foi observado em 48 clientes, que apresentaram tal característica.

Neste estudo, optou-se por utilizar este diagnóstico e não Disfunção Sexual ${ }^{(5)}$, porque ambos são difíceis de diferenciar e Padrões de Sexualidade Alterados é um diagnóstico amplo, do qual a Disfunção Sexual pode fazer parte. Segundo esta mesma autora, o diagnóstico Disfunção Sexual só poderá ser utilizado por profissionais com especialização na área de sexualidade.

A sexualidade refere-se à totalidade da qualidade humana, incluindo os componentes psicológico, emocional, social, cultural, espiritual e não apenas à genitália e seu funcionamento ${ }^{(18)}$. Estes mesmos autores relatam que o enfermeiro deverá fornecer um ambiente terapêutico que conduza à saúde sexual do cliente.

Existe alto risco para este diagnóstico na área de planejamento familiar, pois, dependendo das exigências de determinados métodos contraceptivos, eles podem interferir com as práticas sexuais habituais do casal ${ }^{(11)}$.

Com relação a característica definidora ${ }^{(19)}$, descreveram como "relato de dificuldades", a ausência de libido e a anorgasmia. Quanto as "limitações", apontaram a dispareunia, as etapas do ciclo de desenvolvimento da vida (gravidez, puerpério, menopausa) e a presença de patologias ou terapia medicamentosa que podem afetar a sexualidade.

Neste estudo, observou-se que as ausências de libido, orgasmo e a dispareunia estão intimamente relacionadas a vários fatores descritos na Tabela 6 .

Nas 48 mulheres que apresentaram o diagnóstico de Padrões de Sexualidade Alterados, foi possível identificar a presença de estressores e embora este fator relacionado não tenha sido proposto por Gordon, referencial deste estudo, o memso foi utilizado aqui devido aos inúmeros casos apresentados.

\section{Tabela 6 - Fatores relacionados ao diagnóstico de enfermagem Padrões de Sexualidade Alterados. Serviço de Planejamento Familiar da UNIFESP/EPM. São Paulo, SP 1998}

\section{FNORES RELACIOHDOS}

\section{Estressores}

Ahteração na função e estrutura corporal

Falta de privacidade

Medo (gravidez e DST)

Doença ou tratamento com medicamentos

Relacionamento prejudicado com pessoa significativa

A presença de "estressores" como fator relacionado ao diagnóstico de Padrões de Sexualidade Alterados foi sugerida ${ }^{(5)}$, ao referir que este fator pode ser secundário a problemas de trabalho, preocupações financeiras, conflito de valores e de relacionamento.

A resposta sexual é controlada pelo sistema nervoso autônomo e, na vigência de estresse e ansiedade, torna-se impossível o relaxamento necessário para o êxito na atividade sexual, resultando em experiências insatisfatórias ${ }^{(18)}$.

Durante a entrevista, observou-se que as mulheres relataram fadiga, depressão e ansiedade, decorrentes de estressores que influenciavam a sexualidade.

A "alteração na função e estrutura corporal" foi o segundo fator relacionado a este diagnóstico com $56,2 \%$. A maioria das clientes que havia tido filhos recentemente, relatou problemas referentes à sexualidade, talvez devido a diminuição estrogênica no pós-parto, propiciando alterações na mucosa vaginal e acarretando menor lubrificação e dispareunia. São citados outros autores que referem que este fato ocorre, mesmo na ausência de episiorrafia ${ }^{(20)}$.

Outro fato a ser considerado é a relação da lactação com a atividade sexual, que em virtude das modificações mamárias e endócrinas, ou seja, a sucção ou a manipulação do mamilo faz com que ocorra a liberação de ocitocina, pode intensificar a resposta uterina ao orgasmo. Mas por outro lado, pode ocorrer a ejeção láctea, contribuindo para inibir a erotização no casal ${ }^{(20)}$.

A mulher pode sentir-se menos atraente, no período gestacional e pós-parto, com evidente baixa-estima, o que interfere negativamente na sua sexualidade ${ }^{(21)}$.

Além das modificações orgânicas ocorridas após 0 nascimento de uma criança, a fadiga constitui um grande empecilho para o retorno da atividade sexual prazeirosa, embora este problema possa ser atribuído, também, ao fato da mulher dedicar-se mais ao filho do que ao companheiro, o que pode conduzir a dificuldades no relacionamento e mudanças no comportamento sexual durante 0 período pós-parto, pois o homem pode sentir-se rejeitado e abandonado $^{(12,20)}$.

É importante salientar que as mulheres, no climatério, também podem apresentar dispareunia devida a alterações hormonais e que, associada a outros fatores, pode diminuir a libido e a atividade sexual ${ }^{(22-23)}$.

A "falta de privacidade" representou um percentual de $52,1 \%$ como fator relacionado a este diagnóstico. 0 número de cômodos existentes na casa influenciou profundamente na sexualidade das entrevistadas, que relataram possuir apenas um quarto para toda a família.

0 "medo" de gravidez e de doenças sexualmente transmissíveis representou 35,4\%. Algumas clientes entrevistadas utilizavam métodos contraceptivos, mas o medo de engravidar ainda influenciava a vida sexual do casal. Outras verbalizavam o medo de 
adquirir doenças sexualmente transmissíveis.

As "doenças ou tratamento com medicamentos", que afetaram a sexualidade, apresentaram um índice de ocorrência de 27,1\%. Dentre estas, estão incluídas a insuficiência renal crônica, tratamento com anti-hipertensivos e tratamento com antidepressivos.

0 "relacionamento prejudicado com pessoa significativa", representou apenas $4,2 \%$. As clientes relataram que o companheiro não estava correspondendo ao afeto e que não sentiam prazer no ato sexual.

\section{Estratégias ineficazes de resolução individual}

0 diagnóstico de enfermagem Estratégias Ineficazes de Resolução Individual é definido como comportamento adaptativo e habilidades diminuídas na resolução de problemas para atender as demandas e os papéis da vida. É a incapacidade de controlar situações estressantes da vida para a prevenção/controle, da ansiedade, do medo ou raiva ${ }^{(9)}$.

As características definidoras encontradas para este diagnóstico foram "o relato da presença de problemas estressantes da vida"; "sentimentos de ansiedade, apreensão, receio, depressão ou raiva"; "distúrbio no padrão do sono" e "inabilidade para resolver o problema efetivamente", relatados por 48 clientes $(100,0 \%)$.

0 ser humano encontra agressores durante sua vida os quais desafiam sua capacidade de satisfazer as suas necessidades e de manter o equilíbrio ${ }^{(24)}$. A resposta do indivíduo ao evento estressante depende dos recursos pessoais disponíveis no momento da ocorrência ${ }^{(5)}$.

\section{Nutrição alterada: ingestão maior do que as necessidades corporais}

0 diagnóstico de enfermagem Nutrição Alterada: Ingestão

Maior do que as Necessidades Corporais é definida como sendo o consumo de calorias que excede as necessidades metabólicas ${ }^{(9)}$.

A nutrição deverá ser avaliada em conjunto com outros dados porque existem várias condições que, se forem associadas, poderão interferir na escolha do método contraceptivo, principalmente os hormonais. Clientes que possuem padrões

\section{REFERÊNCIAS BIBLIOGRÁFICAS}

1. Smeltzer SC, Bare BG. Estresse e adaptação. In: Smeltzer SC. Brunner/Suddarth: tratado de enfermagem médico cirúrgica. $7^{\text {th }}$ ed. Rio de Janeiro: Guanabara Koogan; 1994a. p. 99-109. alimentares inadequados, com ingesta excessiva de glicídios, lipídios e carboidratos, poderão desenvolver obesidade e dislipidemia, se correlacionados com vida sedentária e fatores hereditários de certas patologias. Neste aspecto, torna-se interessante, investigar se a paciente está associando o ganho de peso a determinado método contraceptivo pois, muitas clientes acreditam neste acontecimento. A fim de evitar dúvidas, é portanto, recomendável investigar nas consultas subsequentes, a presença de aumento ponderal e se este está relacionado ao uso de anticoncepcionais hormonais ou aumento na ingesta de alimentos mais calóricos.

\section{CONCLUSÃO}

Os diagnósticos de enfermagem com percentuais iguais ou superiores a 45,0\%, em ordem de freqüência, foram:

- Comportamentos para elevar o nível de saúde (100,0\%);

- Potencial para aumento do bem estar espiritual (93,0\%);

- Manutenção da saúde alterada (68,0\%);

- Distúrbio no padrão do sono (66,0\%);

- Risco para controle ineficaz do regime terapêutico $(53,0 \%)$;

- Risco para infecção $(51,0 \%)$;

- Padrões de sexualidade alterados (48,0\%);

- Estratégias ineficazes de resolução individual (48,0\%);

- Nutrição alterada: ingestão maior que as necessidades corporais (47,0\%).

Vale ressaltar que não foi intenção validar as características definidoras dos diagnósticos de enfermagem, encontradas neste estudo, podendo ser objeto de pesquisas futuras. Além disso, é preciso enfatizar que as demais fases do processo de enfermagem, embora tenham sido realizadas na prática assistencial, não foram descritas neste trabalho.

A identificação dos diagnósticos de enfermagem contribuíram para o planejamento e execução de um plano de cuidados direcionado a cada cliente, fundamental para a obtenção de melhor qualidade no atendimento. Ressalta-se a importância da realização de outros estudos em instituições que ofereçam assistência em planejamento familiar, no intuito de observar se os diagnósticos de enfermagem encontrados na população do referido serviço podem ser extensivos a população geral.

2. Barbieri M. 0 enfermeiro na educação de adultos em planejamento familiar. [tese]. São Paulo (SP): Escola de Enfermagem/USP; 1996. 3. Hoga LAK. A mercê do cotidiano da anticoncepção: a mulher seguindo o seu caminho. São Paulo: Escola de Enfermagem/USP; Núcleo de Assistência para o Autocuidado da Mulher; 1996. 
4. lyer PW, Taptich BJ, Bernocchi-Losey D. Processo e diagnóstico em enfermagem. Porto Alegre: Artes Médicas; 1993.

5. Carpenito LJ. Diagnósticos de enfermagem: aplicação à prática clínica. $6^{a}$ ed. Porto Alegre: Artes Médicas; 1997.

6. North American Nursing Diagnosis Association - NANDA. Nursing diagnoses, definitions and classification, 1997/1998. Philadelphia: NANDA; 1996.

7. Marin HF. 0 vocabulário em enfermagem: uma revisão. Acta Paul Enfermagem, set./dez. 1996; 9(3): 68-75.

8. Gordon M. Nursing diagnosis: process and application. $3^{\text {rd }}$ ed. St. Louis: Mosby Year Book; 1994.

9. Gordon M. Manual of nursing diagnosis (1997-1998). St. Louis: Mosby Year Book; 1997.

10. World Health Organization-WHO. Use of body mass index of adults in assessing individual and community nutritional status. Bull World Health Organ 1995; 73(5): 673-80.

11. Reeder SJ, Martin LL, Koniak D. Sexual health and management of family planning. In: Reeder SJ, Martin LL, Koniak D. Maternity nursing: family, newborn and women's health care. $17^{\text {th }}$ ed. Philadelphia: JB Lippincott; 1992. p. 171-216.

12. Czeisler CA, Richardson GS, Martin JB. Distúrbios do sono e do ritmo circadiano. In: Isselbacher KJ, Braunwald E, Wilson JD, Martin JB, Fauci AS, Kasper DL. Harrison: medicina interna. 13ª ed. México (MX): Mc Graw-Hill/Interamericana; 1995. p. 170-8.

13. Silva AB, Lemmi H. Distúrbios do ciclo do sono-vigília. In: Ramos OL, Rotschild HA. Atualização terapêutica $18^{\text {th }}$ ed. São Paulo: Artes Médicas; 1997. p. 707-10.

14. World Health Organization-WHO. Contraceptive method mix: guidelines for policy and service delivery. Geneva: WHO; 1994.
15. Simmons R, Elias $C$. The study of client - provider interactions: a review of methodological issues. Stud Fam Plann 1994; 25(1): 114.

16. Zwelling E. Family planning. In: Nichols FH, Zwelling E. Maternalnewborn nursing: theory and practice. Philadelphia: WB Saunders; 1997. p. 233-74.

17. Ministério da Saúde (BR). Assistência ao planejamento familiar. Brasília (DF): Ministério da Saúde; 1996.

18. Smeltzer SC, Bare BG. Sexualidade humana. In: Smeltzer SC. Brunner/Suddarth: tratado de enfermagem médico cirúrgica. $7^{\text {th }}$ ed. Rio de Janeiro: Guanabara Koogan, 1994b. p. 245-59.

19. Cruz ICF, Brgamasco RB, Belli MA de J, Saito E, Hoga LAK, Martins $\mathrm{R}$ de $\mathrm{CT}$, Florentino LC, Silva IA. Estudo descritivo sobre as características definidoras do diagnóstico de enfermagem: disfunção sexual. Rev Esc Enfermagem USP março 1992; 26(1): 43-64.

20. Yazlle MEHD. Sexualidade e gravidez. Reprodução \& Climatério 1995; 10(2): 56-7.

21. Vitiello N. Sexualidade na gestação e no puerpério. Reprodução \& Climatério 1995; 10 (Supl. 1): 36-7.

22. Silva RO, Fernandes CE, Pereira Filho AS. Climatério: manual de orientação. São Paulo: FEBRASGO; 1995.

23. Lopes GP. Aspectos psicossexuais - síndrome do climatério. In: Lima GR, Baracat EC. Ginecologia endócrina. São Paulo: Atheneu; 1995.

24. Smeltzer SC, Bare BG. Cuidados à mulher com problemas relacionados aos processos fisiológicos. In: Smeltzer SC. Brunner/ Suddarth: tratado de enfermagem médico cirúrgica. $7^{\text {th }}$ ed. Rio de Janeiro: Guanabara Koogan; 1994c. p. 1043-69. 